\title{
DE LA SORTIE DE TERRE À LA MISE SOUS VERRE: LA VÉNUS DE LESPUGUE COMME CHEF-D'CEUVRE DE L'ART MOBILIER PALÉOLITHIQUE. ESSAI DE BIOGRAPHIE MUSÉALE*
}

\author{
FROM DISINTERMENT TO DISPLAY: THE \\ VENUS OF LESPUGUE AS A MASTERPIECE OF \\ PALAEOLITHIC PORTABLE ART. AN ATTEMPT \\ AT A MUSEUM BIOGRAPHY
}

\begin{abstract}
Discovered in August 1922 by the Count of Saint-Périer and given to the laboratory of Paleontology in the National Museum of Natural History of Paris a few months later, the Venus of Lespugue can be seen as a major Palaeolithic work. A lot of theories have tried to explain its meaning and function in Palaeolithic society, but its biography within the museum still remained to be done. This biography will help to examine the situation of the whole of Prehistory within the National Museum of Natural History, to understand the evolution of this institution during the last century, and to reevaluate the rank of masterpiece that has been attributed to the Venus in the new Musée de l'Homme.

Keywords: Venus of Lespugue, musée de l'Homme, masterpiece, museology, prehistory, attempt for a museum biography.
\end{abstract}

\section{Introduction}

Découverte en août 1922 par le comte René de Saint-Périer (1877-1950) et son épouse Suzanne (1890-1978) lors de fouilles de la grotte des Rideaux à Lespugue, en Haute-Garonne, la Vénus de Lespugue ${ }^{1}$ s'impose rapidement

- Adresse pour correspondance: 2, rue Planchat, 75020 Paris, France. Email: marionbbertin@gmail.com.

* Cet article reprend des recherches menées par l'auteur dans le cadre d'un master 2 de recherche en muséologie: M. Bertin, Redéfinir les objets scientifiques en chefs d'œuvre: vie bien vécue ou contre-sens?

${ }^{1}$ Statuette en ivoire de mammouth de 14,7 centimètres. Conservée par le laboratoire d'anthropologie du musée de l'Homme, sous le numéro d'inventaire 19030. 
comme un objet majeur pour l'art paléolithique. Au sein du corpus de statuettes féminines anthropomorphes, retrouvées en nombre à la fin du XIX siècle et dans les premières décennies du $\mathrm{XX}^{\mathrm{e}}$ siècle, elle fait rapidement figure d'exception jusqu'à être qualifiée de reine des Vénus aurignaciennes ${ }^{1}$. Le comte de Saint-Périer reconnaît en elle une synthèse des différentes statuettes trouvées par le passé ${ }^{2}$ lorsqu'il la présente dans un article de la revue L'Anthropologie en 1922, quelques mois après sa découverte. Lorsqu'elle est par la suite donnée au laboratoire de paléontologie du Muséum national d'Histoire naturelle (MNHN), Marcellin Boule (1861-1942), alors tenant de cette chaire, la qualifie quant à lui de plus bel objet paléolithique actuellement connu ${ }^{3}$.

Depuis 1922, la Vénus de Lespugue jouit donc d'une reconnaissance et d'une grande considération de la part des scientifiques du Muséum et d'autres spécialistes de la Préhistoire. Son usage et sa signification ont animé de nombreux débats bien qu'ils demeurent toujours un mystère. Toutefois, ce sont uniquement ces deux éléments qui ont questionné les chercheurs, tout au long du $\mathrm{XX}^{\mathrm{e}}$ siècle et aujourd'hui encore, comme en témoigne la thèse de Raphaëlle Bourrillon portant sur Les Représentations humaines sexuées dans l'art du Paléolithique supérieur européen ${ }^{4}$ qui entreprend un travail d'inventaire et d'interprétation des figures anthropomorphes. Un article d'Arnaud Hurel permet une synthèse de toutes les théories ayant été formulées depuis plus d'un siècle à propos de l'ensemble des Vénus paléolithiques, longuement associées aux théories raciales et ayant favorisé la croyance en l'existence d'une race dite négroïde de Grimaldi ${ }^{5}$. Jusqu'alors la seconde vie de la Vénus de Lespugue, à savoir sa vie muséale, est restée énigmatique. Passée par différents musées et laboratoires en lien avec le Jardin des Plantes, elle illustre pourtant l'évolution des magistères et des institutions liées à l'archéologie préhistorique à Paris. Aujourd'hui conservée par la direction des collections du Muséum national d'Histoire naturelle, elle est toujours attestée comme une des pièces maîtresses de l'art paléolithique ${ }^{6}$ et peut être considérée à certains égards comme la Joconde du musée de l'Homme, où elle est exposée au sein du parcours permanent depuis sa réouverture en octobre 2015, formule qui a été retenue par les membres de l'équipe projet en charge du parcours permanent.

La méthode de la biographie d'objets, inspirée du courant de la microhistoire développée par Giovanni Levi ${ }^{7}$ et Carlo Ginzburg à partir de la notion d'indice ${ }^{8}$, élément parfois minime érigé au rang de pièce à conviction, et par le

\footnotetext{
${ }^{1}$ M. Boule, Les Hommes fossiles, p. 331.

${ }^{2}$ Cf. R. de Saint-Périer, Statuette de femme découverte à Lespugue (Haute-Garonne), p. 373.

${ }^{3}$ Archives du Musée d'Ethnographie du Trocadéro et du Musée de l'Homme, Muséum national d'histoire naturelle: AM 69: Procès-verbaux des Assemblées des professeurs du Muséum, du 20 juin 1918 au 4 mars 1926, $64^{\mathrm{e}}$ volume.

${ }^{4}$ R. Bourrillon, Les Représentations humaines sexuées ... .

${ }^{5}$ Cf. A. Hurel, Des Bushmen en Europe?

${ }^{6} \mathrm{Cf}$. Y. Coppens, L'Ambiguïté des doubles Vénus du Gravettien de France, p. 566.

${ }^{7}$ Cf. G. Levi, On Microhistory.

${ }^{8}$ C. Ginzburg, Signes, traces, pistes.
} 
champ d'étude anglo-saxon consacré à la material culture ${ }^{1}$, se donne pour but de retracer l'histoire d'un objet, sa vie pour poursuivre la métaphore anthropomorphe. La publication de l'ouvrage The Social Life of Things ${ }^{2}$ eut un grand retentissement et permit de penser les objets comme des constructions sociales non figées dans une signification. En France, les travaux de Thierry Bonnot sur les fluctuations de statuts et considérations d'un même objet sont également des sources majeures ${ }^{3}$. La méthode biographique se prête aisément aux collections de musée et permet de mieux comprendre l'histoire de leur constitution, de leur considération, de leur présentation. Les sources sont assez nombreuses et diverses: peuvent ainsi être croisés les archives produites par les musées euxmêmes dont les dossiers d'œuvres, s'il en existe un, et les inventaires, des catalogues d'exposition, la littérature spécialisée, des photographies d'archives. Souvent lacunaires, ces sources présentent des manques qui peuvent devenir significatifs. Elles sont à enrichir également par des témoignages oraux, quand ceux-ci sont possibles, auprès de personnes ayant participé de la vie muséale d'un objet. Cette part d'oralité révèle souvent des éléments absents des archives écrites conservées par les institutions. C'est à partir de l'ensemble de cette documentation que la biographie de la Vénus de Lespugue a pu être ébauchée, dans la limite des faits retenus par la mémoire et leurs lacunes.

Quelles sont les différentes institutions ayant conservée la Vénus de Lespugue? Quand et comment a-t-elle été exposée? En vue de quels discours? À l'inverse, à quels moments a-t-elle été sortie des vitrines et remise en réserves? Pour quelles raisons? Quelles positions et théories, défendues par les scientifiques du MNHN, a-t-elle illustrées au sein du musée? Celui-ci s'est-il ou non placé au cœur des débats doctrinaux? Retracer la biographie muséale de la Vénus de Lespugue permettra de répondre à ces différentes questions en ouvrant de nouvelles perspectives de recherche. Elle aidera à mieux comprendre les différentes prérogatives d'exposition et de gestion des collections rencontrées par les équipes du MNHN au cours du XX ${ }^{\mathrm{e}}$ siècle. Elle esquissera en outre la considération et la place de la discipline préhistorique au sein du MNHN. La dialectique entre mémoire et oubli, la place des archives tant orales qu'écrites, et leurs ponctuelles divergences, autour de l'histoire des collections sera omniprésente. Finalement, par cette biographie se poseront les questions des interactions parfois tumultueuses entre science et musée ${ }^{4}$ et du statut de chefd'œuvre acquis par certains objets aujourd'hui. Dans le cas d'objets scientifiques ayant servi à l'élaboration de disciplines scientifiques, est-ce là le reflet d'une crise mettant en cause la place même de la science au sein de l'institution? Ou n'est-ce que l'aboutissement d'un processus plus long, montrant la difficile

\footnotetext{
${ }^{1}$ La culture matérielle est définie par C. Tilley, Objectification, p. 61, comme a particular way of understanding the relationship between subjects and objects [Un moyen particulier de comprendre la relation entre les sujets et les objets].

${ }^{2}$ Cf. The Social Life of Things, (éd.) A. Appadurai.

${ }^{3}$ T. Bonnot, La Vie des objets \& T. Bonnot, L'Attachement aux choses.

${ }^{4}$ Telle que l'ont montré les recherches au sujet de l'anatomie comparée au MNHN menées par C. Crémière, La Science au musée.
} 
situation de la science dans l'espace muséal, plus encore présent concernant la discipline préhistorique?

\section{Avant le musée}

Médecin et biologiste, René Poilloüe, comte de Saint-Périer, se passionne pour la préhistoire en plein développement et réalise des fouilles sur plusieurs sites datés du Paléolithique supérieur dans le sud-ouest de la France. En 1911, il réalise une première campagne de fouilles dans la grotte des Rideaux à Lespugue, en Haute-Garonne, nommée comme telle en raison d'un réseau de stalactites sur la paroi nord de la caverne. Ces fouilles sont arrêtées un temps pendant la Première Guerre mondiale. C'est à cette époque que le comte de Saint-Périer rencontre Suzanne Raymonde François qu'il épouse en 1920. Ensemble, ils reprennent les fouilles de la grotte des Rideaux en $1922^{1}$.

La stratigraphie du site est jugée probable, bien qu'incertaine ${ }^{2}$, et rendue avec quelques précisions par le comte de Saint-Périer ${ }^{3}$. La grotte des Rideaux fut occupée à différentes époques et donc plusieurs fois remaniée: le couple Saint-Périer mit au jour des vestiges médiévaux, gallo-romains, des tessons émaillés plus récents. Concernant la couche la plus ancienne, dont le foyer était situé à cinq mètres de l'entrée de la grotte ${ }^{4}$, ils la datèrent de l'Aurignacien supérieur ${ }^{5}$. Les restes animaux correspondant à l'âge $d u$ renne 6 et le type d'outillage mis au jour justifiaient en effet ce rapprochement. Le 9 août, alors que les fouilles se terminent, un des ouvriers donne un dernier coup de pioche et découvre celle qui sera nommée la Vénus de Lespugue, à seulement quinze centimètres de profondeur, mise à l'écart dans une zone d'ombre de la grotte. Le heurt, à la fois heureux dans l'invention de la statuette et malencontreux dans son immédiate conséquence, brise l'objet en deux parties, auxquelles s'ajoutent neuf fragments retrouvés à proximité dans la terre. Devenu très fragile, il est un peu plus abîmé à chaque manipulation ${ }^{7}$. Son inventeur nous livre une description précise et approfondie de l'objet dans un article où il annonce sa découverte:

La statuette est sculptée en ronde-bosse dans une défense de mammouth. Elle mesure 0,147 mètre de hauteur; la tête est petite et ovalaire, le visage ne porte

\footnotetext{
${ }^{1}$ Cette notice biographique s'inspire de celle réalisée par Ch. Normand in: Le Muséum de Toulouse et l'invention de la Préhistoire, pp. 77-78.

${ }^{2}$ L. Pales, Les Ci-devant Vénus stéatopyges aurignaciennes, pp. 217-261, signale en effet un manque de rigueur dans ces fouilles, mais juge la stratigraphie plausible en regard des objets mis au jour.

${ }^{3}$ Cf. R. de Saint-Périer, Statuette de femme découverte à Lespugue (Haute-Garonne). Les détails évoqués par la suite sur les fouilles de la grotte des Rideaux sont issus de cet article.

${ }^{4}$ Le foyer renvoie au vocabulaire du début du $\mathrm{XX}^{\mathrm{e}}$ siècle en matière de fouilles; il désigne la couche archéologique. Précision donnée par D. Vialou, Lespugue, femme ou Vénus?, p. 37.

${ }^{5}$ C'est-à-dire environ 36 000-34 000 Before Present (= BP).

${ }^{6}$ Proposition de classement chronologique effectuée par Édouard Lartet pour les périodes les plus anciennes de l'histoire de l'humanité, en fonction des restes animaux majoritairement trouvés en fouilles. Voir notamment H. Christy \& É. Lartet, Sur des figures d'animaux gravées ou sculptées ... .

${ }^{7}$ Cf. R. de Saint-Périer, Statuette de femme découverte à Lespugue (Haute-Garonne), p. 364.
} 
pas de traits distincts. Des lignes parallèles, figurant les cheveux, descendent en avant sur les trois quarts de la face et en arrière jusqu'au niveau des omoplates. Le cou est mince, le thorax maigre, d'énormes seins en forme d'outres pendent jusqu'au ventre, petit et bombé, qu'ils repoussent en avant. Les bras, qui reposent sur les seins, sont détachés du tronc dans leur tiers inférieur, ce qui suppose chez le sculpteur une grande habileté technique. La région fessière est remarquable par son ampleur: les fesses sont rejetées latéralement et aplaties; elles présentent, à leur partie inférieure, une bride surmontée d'une petite éminence qui me paraît correspondre à l'indication d'une fistule coccygienne. Les cuisses présentent également une large saillie latérale, les jambes sont courtes et les pieds à peine indiqués. La fracture antérieure intéresse malheureusement la région pubienne, ce qui ne nous permet pas de savoir si les organes génitaux avaient été figurés. Il faut noter la présence, au-dessous des fesses, d'un singulier vêtement, en forme de pagne triangulaire, qui paraît constitué par des bandes tressées, terminées par une frange. ${ }^{1}$

Cette découverte intervient dans le cadre d'autres trouvailles de figurations féminines en matières dures animales ou en pierre. La première à être exhumée est la Vénus impudique, mise au jour par le marquis de Vibraye (1809-1878) en 1864 sur le site de Laugerie-Basse aux Eyzies de Tayac, en Dordogne, et nommée ainsi en raison de son triangle pubien profondément gravé2. L'appellation de Vénus s'impose rapidement, bien qu'il soit difficile d'attribuer à un scientifique cette paternité. Un consensus se fait néanmoins autour de la personne du marquis de Vibraye, à la suite de cette découverte ${ }^{3}$. La Vénus impudique est la première à pouvoir être décrite comme femme paléolithique nue statufiée 4 avant que d'autres découvertes n'interviennent entre la fin du $\mathrm{XIX}^{\mathrm{e}}$ siècle et le début du $\mathrm{XX}^{\mathrm{e}}$ siècle à travers toute l'Europe, du nord de l'Espagne à l'Europe de l'est. Selon le Dictionnaire de la Préhistoire établi par Denis Vialou, la dénomination Vénus est un terme générique servant à désigner moins d'une centaine de figurations féminines paléolithiques le plus souvent sculptées en bas-relief sur paroi rocheuse ou en ronde-bosse $e^{5}$. Certaines des

\footnotetext{
${ }^{1}$ R. de Saint-Périer, La Statuette féminine de Lespugue (Haute-Garonne), pp. 82-83.

${ }^{2}$ Statuette en ivoire de mammouth de huit centimètres de hauteur, également conservée dans les collections du MNHN.

${ }^{3}$ Cf. A. Hurel, Des Bushmen en Europe?, pp. 306-310. Voir également L. Pales, Les Ci-devant Vénus stéatopyges aurignaciennes, p. 218.

${ }^{4}$ L. Passemard, Les Statuettes féminines paléolithiques dites Vénus stéatopyges, p. 4 (le texte original est lui-même en italique).

${ }^{5}$ La Préhistoire. Histoire et dictionnaire, (éd.) D. Vialou, p. 1369.
} 
Vénus reçoivent des noms en fonction de leur forme, telle la Vénus impudique, mais dans le cas de la Vénus de Lespugue, c'est le lieu de son invention qui a été privilégié par le comte de Saint-Périer. Dès le premier article qu'il publie au sujet de sa découverte dans la célèbre revue L'Anthropologie, la statuette prend l'appellation qui restera la sienne jusqu'à nos jours. L'auteur utilise toutefois l'expression «Vénus de Lespugue» entre guillemets et la désigne plutôt comme la statuette féminine de Lespugue, comme l'indique le titre de l'article ${ }^{1}$. $\mathrm{Au}$-delà de leurs appellations, ces statuettes sont qualifiées de stéatopyges par Édouard Piette (1827-1906), après ses découvertes de huit figurines féminines sur le site de Brassempouy, dans les Landes, entre 1894 et $1897^{2}$. À une époque où les populations préhistoriques étaient considérées comme relevant d'un très haut degré de primitivité, les premières controverses portent sur la datation et l'ancienneté plausible de tels objets ${ }^{3}$.

Par la suite, et pendant plusieurs décennies, les réflexions s'intéressent avant tout à leur aspect physique et leur véracité plausible. Proche de l'archéologie et de la géologie, la discipline paléontologique se développe au cours de la seconde moitié du XIX ${ }^{\mathrm{e}}$ siècle alors que les découvertes de vestiges préhistoriques se multiplient. La paléontologie humaine, aussi nommée paléoethnologie, s'inspire toutefois des réflexions menées en anthropologie et se spécialise dans la reconstitution de la réalité physique et sociale des populations préhistoriques. La méthode comparatiste, établissant des similitudes entre les premiers habitants de l'Europe et les sociétés exotiques contemporaines, est reprise dans ce dessein et érigée comme modèle à suivre. L'anthropologie culturelle accompagne alors la paléontologie ${ }^{4}$. Fortement liées à cette époque, ces deux disciplines se nourrissent l'une et l'autre autour de la question raciale et de ses débats. Comme l'a souligné dans sa thèse Raphälle Bourrillon, ces premières découvertes de représentations humaines vont faire davantage l'objet d'une approche morphologique et anthropologique que véritablement archéologique et stylistique ${ }^{5}$ : la forme supplant [e] le sens ${ }^{6}$, telle que l'illustre la description donnée par le comte de Saint-Périer dans le Bulletin de la Société préhistorique de France.

À la suite de ses découvertes sur le site de Brassempouy, Édouard Piette développe une théorie rapprochant les anciennes populations européennes de la race $^{7}$ Boschimane ${ }^{8}$, peuple vivant en Afrique du Sud. Leurs formes généreuses frapperont l'imagination des préhistoriens qui leur donneront tout de suite le

\footnotetext{
${ }^{1}$ Cf. R. de Saint-Périer, Statuette de femme découverte à Lespugue (Haute-Garonne), p. 379.

${ }^{2}$ Cf. É. Piette, Station de Brassempouy et statuettes humaines de la période glyptique \& É. Piette, Races humaines de la période glyptique.

${ }^{3} \mathrm{Cf}$. D. Vialou, Lespugue, femme ou Vénus?

${ }^{4}$ Cf. A. Hurel, Des Bushmen en Europe?, p. 295.

${ }^{5}$ Cf. R. Bourrillon, Les Représentations humaines sexuées ..., p. 16.

${ }^{6}$ Cf. A. Hurel, Des Bushmen en Europe?, p. 295.

${ }^{7}$ Ce terme est employé dans le sens classificatoire qu'il pouvait recouvrir au XIX ${ }^{\mathrm{e}}$ siècle.

${ }^{8}$ Les Sans constituent un ensemble de peuples autochtones vivant en Afrique australe. Le terme San tend de nos jours à remplacer Bochiman (homme du bush), utilisé durant la période coloniale.
} 
nom de «Vénus» dans une référence assumée aux femmes khoisan". Tel que l'écrit le comte de Saint-Périer dans un autre article de la revue L'Anthropologie:
Il me semble en effet que les caractères anatomiques de cette statuette sont trop nettement semblables à ceux des races stéatopyges actuelles pour qu'il soit possible de voir dans cette représentation une convention purement artistique ou religieuse, comme cela fut soutenu. ${ }^{2}$

Il est indéniable pour le comte de Saint-Périer qu'à l'époque aurignacienne, une race du groupe de quelques races africaines actuelles vivait en Europe ${ }^{3}$. L'existence d'une race négroïde de Grimaldi ${ }^{4}$ est soutenue par de nombreux savants au premier rang desquels Marcellin Boule. Dans son article du Bulletin de la Société préhistorique de France, le comte de Saint-Périer évoque d'ailleurs plusieurs rapprochements ethnographiques entre la statuette qu'il vient de mettre au jour et les populations actuelles. La Vénus de Lespugue se distingue rapidement de ses compagnes sculptées par son caractère exceptionnel. En effet, elle est la seule à être en partie vêtue, particularité unique reconnue dès son invention ${ }^{5}$. Le comte de Saint-Périer rapproche le pagne dont est revêtue la Vénus avec ceux portés par les femmes de populations Madi, Soudanaises, Cafres ou Dinka, ou encore des feuilles dont se servent les femmes Obongos $^{6}$. Il fait également plusieurs hypothèses sur cet habit: symbolique de la situation maritale de la femme comme en Ouganda ou attaché bas afin de masquer ses parties génitales comme aux Philippines ${ }^{7}$.

Le comte de Saint-Périer reste à l'écart des autres débats concernant les significations des Vénus paléolithiques et leurs sources d'inspiration ${ }^{8}$. Déessesmères? Femmes soumises? Symboles de la fécondité? Représentation d'un idéal de corps féminin ${ }^{9}$ ? La possibilité d'une forme d'art paléolithique est assez rapidement diffusée parmi les préhistoriens grâce à la découverte de gravures et petites sculptures. L'expression art mobilier devient canonique afin de désigner les représentations artistiques présentes sur des supports trans-

${ }^{1}$ Cf. A. Hurel, Des Bushmen en Europe?, p. 293.

${ }^{2}$ Cf. R. de Saint-Périer, Statuette de femme découverte à Lespugue (Haute-Garonne), p. 370.

${ }^{3}$ Cf. R. de Saint-Périer, Statuette de femme découverte à Lespugue (Haute-Garonne), p. 379.

${ }^{4}$ Selon l'expression de René Verneau (1852-1938) à la suite de ses découvertes de la grotte des Enfants à Balai Rossi en Italie en 1901. M. Boule, Les Hommes fossiles, tenta d'établir les différents types physiques ayant vécu au cours du Paléolithique supérieur.

${ }^{5}$ Cf. R. de Saint-Périer, La Statuette féminine de Lespugue (Haute-Garonne).

${ }^{6}$ Cf. R. de Saint-Périer, Statuette de femme découverte à Lespugue (Haute-Garonne), pp. 370-371. Toutes les populations citées sont africaines.

${ }^{7}$ Cf. R. de Saint-Périer, Statuette de femme découverte à Lespugue (Haute-Garonne), p. 371.

${ }^{8}$ Cf. R. de Saint-Périer, Statuette de femme découverte à Lespugue (Haute-Garonne), p. 374: Dans l'état actuel de nos connaissances, nous devons envisager les statues paléolithiques comme des manifestations artistiques qui comportent seulement des rapprochements d'ordre morphologique et ethnique.

${ }^{9}$ Cf. G.-H. Luquet, Les Vénus paléolithiques. Pour un résumé des diverses propositions et leurs tenants: $\mathrm{H}$. Delporte, L'Image de la femme dans l'art préhistorique \& D. Vialou, Lespugue, femme ou Vénus? 
portables ou mobiliers ${ }^{1}$. Mais les débats autour du sens de ces objets n'aboutissent pas au même consensus. Deux cercles de pensées, surtout marqués au XIX ${ }^{\mathrm{e}}$ siècle, s'opposent autour de la destination de l'art préhistorique: art magique ou art pour l'art. Ces débats rendent compte de la confrontation de savants de diverses disciplines, médecins ou historiens de l'art et des religions.

En raison de mauvaises chronologies des sols établies lors des premières fouilles, causées par des analogies avec des éléments trouvés à leurs côtés puis par analyse stylistique des différentes statuettes, les Vénus ont longtemps été datées de la période aurignacienne, au sens que le Professeur Breuil attache à cette appellation ${ }^{2}$. André Leroi-Gourhan (1911-1986) est parmi les premiers à contester cette attribution ${ }^{3}$, dont la longue erreur est mise en valeur dans un article du professeur Léon Pales (1905-1988) publié en $1972^{4}$. En réalité, on date actuellement ces séries de statuettes de la période gravettienne, c'est-àdire entre 31000 et 22000 avant le présent. Tel que l'a énoncé Raphaëlle Bourrillon, les Vénus forment aujourd'hui le fossile directeur de cette période ${ }^{5}$ et permettent ainsi de dater nettement des sites de fouilles.

\section{Vie muséale de la Vénus}

Fondamentalement positive, la science préhistorique s'est développée en parallèle des musées qui lui étaient dédiés, permettant la conservation des objets d'études sur lesquels fonder son savoir. Le musée tient une place majeure dans le processus patrimonial et l'écriture des disciplines ${ }^{6}$. Véritable science de musée, l'archéologie préhistorique est indissociable des établissements de conservation. C'est également par et grâce aux musées que la préhistoire s'institutionnalise et qu'elle obtient reconnaissance et pérennité, bien avant d'être enseignée à l'université ${ }^{7}$. Au MNHN, elle connait des débuts d'abord difficiles avec l'imposition d'une chaire de paléontologie par décret impérial en $1853^{8}$ et la difficile intégration des collections données par Jacques Boucher de Perthes $(1788-1868)^{9}$. La question des origines de l'humanité se trouve sans cesse partagée entre les différentes chaires, paléontologie et anthropologie, ce qui est également le cas pour les collections préhistoriques. L'approche y est

\footnotetext{
${ }^{1}$ Le Musée de l'Homme: itinéraire, p. 86.

${ }^{2}$ Cf. L. Passemard, Les Statuettes féminines paléolithiques dites Vénus stéatopyges, p. 56. On doit à l'abbé Henri Breuil (1877-1961) la mise en évidence de l'Aurignacien, entre 43000 et 29000 BP, en tant que première culture européenne de l'Homme moderne du Paléolithique supérieur.

${ }^{3}$ Cf. A. Leroi-Gourhan, Préhistoire de l'art occidental, p. 113.

${ }^{4} \mathrm{Cf}$. L. Pales, Les Ci-devant Vénus stéatopyges aurignaciennes. Cet article est également intéressant en ce qu'il dément la plupart des théories construites autour de ces statuettes paléolithiques: leur dénomination de Vénus, leur stéatopygie, les théories formelles d'André Leroi-Gourhan, les groupes définis par Édouard Piette, et enfin leur datation.

${ }^{5}$ Cf. R. Bourrillon, Les Représentations humaines sexuées ..., , p. 19.

${ }^{6}$ Cf. D. Poulot, Patrimoine et musées, p. 11

${ }^{7}$ Cf. A. Hurel, Aux marches du Palais, la place de la Préhistoire au Trocadéro.

${ }^{8}$ Cf. M.-T. Vénec-Peyré, La Difficile Naissance de la chaire de Paléontologie ... .

${ }^{9} \mathrm{R}$. Orliac, L'invention de la préhistoire par les objets.
} 
avant tout naturaliste ${ }^{1}$. En 1910 est créé l'Institut de Paléontologie Humaine (IPH) sur l'initiative du professeur Marcellin Boule, de l'abbé Henri Breuil et du prince Albert I ${ }^{\mathrm{er}}$ de Monaco (1848-1922). Ce lieu unique permet une étude totale de l'homme préhistorique alliant approches physique, culturelle et environnementale, et vient compléter le MNHN où la jeune discipline préhistorique se développe dans le giron d'autres sciences plus établies.

La Vénus de Lespugue est envoyée comme don au MNHN, plus précisément au laboratoire de paléontologie, dont la chaire est alors occupée par Marcellin Boule, dès le mois de décembre $1922^{2}$. Ce don est immédiatement acclamé et la Vénus est caractérisée de plus bel objet paléolithique actuellement connu ${ }^{3}$. Cette donation intervient bien avant la promulgation de la loi Carcopino en date du 27 septembre $1941^{4}$. Les objets trouvés en fouilles avant cette date étaient dépositaires de leurs inventeurs, qui pouvaient choisir de les conserver personnellement ou désigner eux-mêmes un donataire. D'autres lieux de conservation auraient pu être envisagés. Des objets provenant de la grotte des Rideaux ont ainsi été donnés au musée de Lespugue par le couple Saint-Périer, mais ils n'ont pas l'importance de la Vénus ${ }^{5}$. Le Musée d'archéologie nationale de Saint-Germain-en-Laye, dont la raison d'être est de mettre en valeur une préhistoire nationale, conserve d'autres objets découverts par la suite par les Saint-Périer, mais ceux-ci proviennent tous de fouilles plus tardives de la grotte d'Isturitz, dans les Pyrénées-Atlantiques. Ce don au MNHN permet d'interroger une filiation intellectuelle entre le couple Saint-Périer et Marcellin Boule. Les deux scientifiques partageaient en effet un attrait pour les théories de Piette en rapprochant ces statuettes féminines de populations contemporaines. La correspondance postérieure échangée entre les Saint-Périer et le couple Boule indique une bienveillance certaine entre $\mathrm{eux}^{6}$.

Cette entrée au musée est non négligeable pour la conservation physique de la statuette, très fragilisée après le coup de pioche qui avait permis sa découverte. Neuf fragments d'ivoire ont été recueillis par le comte de SaintPérier à proximité de la Vénus dans la grotte des Rideaux. Arrivée au MNHN, elle est confiée à M. Barbier, chef de l'atelier de moulage du laboratoire de Paléontologie, [qui] fut chargé du travail délicat de nettoyage, de consoli-

\footnotetext{
${ }^{1}$ Cf. A. Hurel, Les Préhistoriques sous l'œeil des naturalistes.

${ }^{2}$ Archives du Musée d'Ethnographie du Trocadéro et du Musée de l'Homme, Muséum national d'histoire naturelle: AM 69: Procès-verbaux des Assemblées des professeurs du Muséum, du 20 juin 1918 au 4 mars 1926, $64^{\mathrm{e}}$ vol.

${ }^{3}$ Archives du Musée d'Ethnographie du Trocadéro et du Musée de l'Homme, Muséum national d'histoire naturelle: AM 69: Procès-verbaux des Assemblées des professeurs du Muséum, du 20 juin 1918 au 4 mars 1926 , $64^{\mathrm{e}}$ vol.

${ }^{4}$ Journal officiel de la République française, lois et décrets, en date du 15 octobre 1941.

${ }^{5}$ Cf. P. Foucher \& C. San Juan, Les Industries solutréennes de l'abri des Harpons ... .

${ }^{6}$ Archives de l'Institut de Paléontologie Humaine: Correspondance du comte de Saint-Périer avec Marcellin Boule (1936-1939). Les lettres sont rédigées pour la plupart par Suzanne de Saint-Périer qui emploie un vocabulaire empreint d'affection à l'égard du couple Boule.
} 
dation, de restauration ${ }^{1}$ et, s'en [étant] acquitté avec un soin et un zèle ${ }^{2}$, a permis la préservation la Vénus de Lespugue (cf. Illustration $\mathrm{n}^{\circ} 1^{3}$ ).

Après son don, la Vénus de Lespugue est enregistrée dans les collections du laboratoire de paléontologie au numéro 1922-21. La description indique statuette féminine stéatopyge en ivoire trouvée par M. de Saint-Périer à Lespugue (Haute-Garonne) ${ }^{4}$. Une mention dans la marge signale musée de l'Homme : le registre de cession des collections du laboratoire de paléontologie fait état du transfert de la Vénus en faveur du musée de l'Homme, le 4 mars $1939^{6}$. Elle porte le numéro de cession 1939-1. Paul Rivet (1876-1958), nommé à la chaire d'anthropologie du Muséum et à la tête du musée d'ethnographie du Trocadéro en 1928, entreprend une réforme des disciplines liées à l'étude de l'Homme au Jardin des Plantes. Il développe également un projet de musée en lieu et place du musée d'ethnographie du Trocadéro qui ferme ses portes en 1935. Rivet impose progressivement une hégémonie dans tout le champ de compétences en matière d'ethnologie et y réunit l'anthropologie tant culturelle que physique. L'ethnologie s'entend [alors] comme cette méta-science qui coiffe toutes les disciplines ${ }^{7}$, revenant à une discipline généraliste telle que pensée par Armand de Quatrefages (1810-1892). Ouvert en 1938, le musée de l'Homme intègre des laboratoires en ses murs, permettant la collaboration étroite entre recherche et diffusion. Comme l'a souligné l'anthropologue Benoît de l'Estoile, [m]anifestation, laboratoire et école, le musée de l'Homme a incarné la nouvelle science de l'Homme, l'ethnologie, dont il se voulait à la fois le temple et le principal outil, consacré à la production et à la transmission du savoir ${ }^{8}$. Le parcours permanent du musée permet d'illustrer les théories de Rivet au sujet de l'unité de l'esprit et de la race humaine. Un parcours unique crée une trame narrative passant du biologique vers le culturel, de l'universel vers les aspects particuliers d'une aire géographique, de l'anthropologie physique à l'ethnographie des continents et à la préhistoire ${ }^{9}$. Toutefois, toutes les disciplines ne sont pas égales dans leur association au musée de l'Homme. Ainsi en est-il des sciences liées à la préhistoire, rattachées à l'ethnologie après la suppression de la chaire de paléontologie au moment du départ à la retraite de Marcellin Boule en janvier

\footnotetext{
${ }^{1}$ Cf. R. de Saint-Périer, Statuette de femme découverte à Lespugue (Haute-Garonne), p. 364.

${ }^{2}$ Cf. R. de Saint-Périer, Statuette de femme découverte à Lespugue (Haute-Garonne), p. 364.

${ }^{3}$ Toutes les illustrations ont été placées à la fin de l'article.

${ }^{4}$ Archives du laboratoire de paléontologie du Muséum national d'Histoire naturelle: Registre d'inventaire des collections paléontologiques.

${ }^{5}$ Archives du laboratoire de paléontologie du Muséum national d'Histoire naturelle: Registre d'inventaire des collections paléontologiques.

${ }^{6}$ Archives du laboratoire de paléontologie du Muséum national d'Histoire naturelle: Registre de cession des collections paléontologiques.

${ }^{7}$ Cf. C. Laurière, Paul Rivet (1876-1958), le savant et le politique, p. 481.

${ }^{8}$ Cf. B. de l'Estoile, Le Goût des Autres, pp. 39-40.

${ }^{9}$ Cf. F. Grognet, Le concept de musée ..., p. 383.
} 
1936². Le déplacement des collections préhistoriques du MNHN en faveur du laboratoire du musée d'ethnographie du Trocadéro est annoncé lors de l'assemblée des professeurs-administrateurs du 16 janvier 1936. Elles sont rattachées à la chaire d'étude des hommes actuels et des hommes fossiles tenue par Rivet. Au sein des différents départements du musée de l'Homme, celui de paléo-ethnologie reçoit les matériaux lithiques, les ossements fossiles et objets en matière dure animale revenant aux collections d'anthropologie ${ }^{2}$.

Il n'est toutefois pas certain que la Vénus de Lespugue ait réellement pris le chemin du musée de l'Homme en 1939. À partir de 1938 et face à l'imminence d'un nouveau conflit mondial, les musées se préparent à faire face et envisagent des plans d'évacuation des collections. Celui du musée de l'Homme est mis en place au cours de l'automne 1938 et envisage le déplacement des collections les plus fragiles, qui sont stockées à la Banque de France pendant la durée du conflit. Des listes d'urgence sont rédigées par chacun des départements du musée en vue du déplacement des collections dès le 26 septembre $1938^{3}$. Parmi les objets du laboratoire d'anthropologie, restes osseux les plus anciens ou les plus rares, tels que les crânes de Cro-Magnon, on compte la Vénus de Lespugue, ajout manuscrit sur une liste dactylographiée conservée à présent à la Bibliothèque centrale du MNHN. La Seconde Guerre mondiale entraîne de plus la fermeture complète du musée de l'Homme à partir de septembre 1939, dans un but de protection des collections. Toutefois à la demande de Rivet, il est à nouveau ouvert au public en novembre 1939.

Le numéro patrimonial actuel de la Vénus de Lespugue au sein du laboratoire d'anthropologie du musée de l'Homme est le 19030. La description dans le registre d'inventaire des collections indique statuette féminine en ivoire de mammouth trouvée dans la grotte des Rideaux à Lespugue (Haute-Garonne) par M. et Mme de Saint-Périer (Moulage). Don de Monsieur de Saint-Périer ${ }^{4}$. Dès sa découverte, la Vénus de Lespugue a été reproduite et dupliquée: des moulages ont été réalisés par le laboratoire de paléontologie du MNHN afin d'être diffusés auprès des différents organes d'études de l'Homme paléolithique. L'un des plus connus est celui réalisé à la demande de Marcellin Boule proposant une version restaurée de la Vénus au deux tiers de sa taille normale . $^{5}$ Les archives du laboratoire de paléontologie conservent un registre rendant compte des sorties d'objets et de moulages du laboratoire. Bien que plus tardives, y sont dénombrées seize mentions de dons ou ventes de fac-similés de

${ }^{1}$ Cf. A. Hurel, Quelle place pour la Préhistoire au Palais du Trocadéro?

${ }^{2} \mathrm{Cf}$. A. Hurel, Aux marches du Palais, la place de la Préhistoire au Trocadéro. Avant la fermeture du musée d'ethnographie du Trocadéro, un laboratoire de Préhistoire fut mis en place en 1932 par Harper Kelley (18961962), puis l'année suivante une section d'exposition dédiée spécifiquement à l'art rupestre africain, présentant les relevés établis par Kelley et l'abbé Henri Breuil.

${ }^{3}$ Archives du Musée d'Ethnographie du Trocadéro et du musée de l'Homme, Muséum national d'Histoire naturelle: 2 AM 1 I4c, Mouvements des collections; département des services techniques.

${ }^{4}$ Archives du laboratoire d'anthropologie du MNHN: Registre d'inventaire des collections anthropologiques, vol. 2 (numéros 5000 à 21 162).

${ }^{5}$ Cf. R. de Saint-Périer, Statuette de femme découverte à Lespugue (Haute-Garonne), p. 365. 
la Vénus de Lespugue entre 1950 et $1960^{1}$. La plupart est entrée ensuite dans les collections du musée de l'Homme et il est plus que probable que dès la découverte de la statuette, le laboratoire d'anthropologie, sous la direction de René Verneau entre 1879 et 1928, ait reçu un exemplaire. Un second registre d'inventaire, faisant état des entrées d'objets par année, est conservé au laboratoire d'anthropologie ${ }^{2}$. La Vénus de Lespugue est inscrite à l'année 1926, sous le numéro 1926.5 sans qu'il soit toutefois fait mention de moulage. L'hypothèse qui peut être formulée est donc qu'un moulage est entré dans les collections du laboratoire d'anthropologie en 1926 et qu'il a reçu par la suite le numéro patrimonial définitif 19030. Entré dans les mœurs par le personnel du laboratoire et sans autre mention dans les registres de l'arrivée de la véritable Vénus de Lespugue dans les collections en 1939, il a perduré dans l'usage jusqu'à aujourd'hui. Le moulage acquis en 1926 et les autres datant des années 1950 n'ont pas été retrouvés. D'après ce qui a été mentionné sur les déplacements des collections sous la responsabilité du MNHN entre les années 1920 et les années 1940, la date d'arrivée dans les collections du laboratoire d'anthropologie ne peut être certifiée.

D'autres musées ou lieux de recherche ont également reçu de tels moulages, tel que l'IPH. Deux moulages de la Vénus de Lespugue sont entrés dans ses collections en 1923, une version dans son état détérioré et une version restaurée. Aucune reproduction de la Vénus de Lespugue n'a pourtant été retrouvée dans les collections qui en conservaient un exemplaire. Ces moulages devaient être réalisés en plâtre, matériau couramment employé pour les reproductions utiles aux musées d'archéologie ${ }^{3}$, mais la version restaurée à la demande de Marcellin Boule intègre également de la plastiline ${ }^{4}$. Des photographies de l'atelier de Pablo Picasso (1881-1973) aux Grands-Augustins à Paris prises par Brassaï en 1943 rendent compte de la présence de moulages de la Vénus de Lespugue dans le musée personnel de l'artiste, ce qui atteste de la diffusion de ces répliques.

Les avis divergent quant aux dates de l'exposition de la statuette dans les galeries permanentes du musée de l'Homme. Selon les éléments communiqués par Odile Romain, chargée de collections en préhistoire au MNHN, la véritable Vénus de Lespugue n'aurait jamais été exposée en tant que telle dans le parcours permanent du musée de l'Homme avant sa réouverture en $2015^{5}$. Par manque de moyens techniques et financiers, et par mesure de conservation préventive, le musée aurait toujours eu recours à des moulages. Toutefois, les recherches menées par Fabrice Grognet sur l'histoire du musée de l'Homme lui ont permis d'établir que la Vénus de Lespugue était exposée au sein de la galerie d'anthropologie, dans la deuxième salle consacrée à la découverte des races

\footnotetext{
1984).

${ }^{1}$ Archives du laboratoire de paléontologie du MNHN: Registre de sortie d'objets et de moulages (1948-

${ }^{2}$ Archives du laboratoire d'anthropologie du MNHN: Registre d'entrée par date en collections d'anthropologie, vol. 2 .

${ }^{3}$ Cf. A. Hurel, Faire entrer la Préhistoire au musée, p. 163.

${ }^{4}$ Cf. R. de Saint-Périer, Statuette de femme découverte à Lespugue (Haute-Garonne), p. 364.

${ }^{5}$ Communication personnelle d'Odile Romain, le 4 avril 2016.
} 
fossiles et actuelles, selon la formule de Paul Rivet. Des objets préhistoriques y figuraient, dont la célèbre Vénus, aux côtés d'éléments d'art pariétal, ainsi que du moulage de la Vénus Hottentote, rappel des premières théories raciales qui avaient pu les associer ${ }^{1}$. Philippe Mennecier, ancien responsable des collections anthropologiques au musée de l'Homme, soutient cette idée et se souvient avoir pu voir la Vénus de Lespugue au sein des galeries permanentes, présentée dos à un miroir afin d'en observer toutes les faces ${ }^{2}$. Mais à partir de quelle date at-elle exposée? Était-ce la véritable Vénus?

Une exposition majeure dans la vie de la Vénus de Lespugue est celle de 1965 consacrée aux Chefs-d'œuvre du musée de l'Homme. Elle fut financée par la Société des Amis du musée de l'Homme, présidée à cette époque par la baronne de Rothschild qui permit des fastes années à l'association. Peut-on ainsi supposer des dispositions particulières à cette occasion afin de protéger la Vénus de Lespugue et de la présenter au public? Les archives restent silencieuses à ce sujet et les quelques photographies de la salle d'exposition ne permettent pas d'y distinguer la Vénus de Lespugue. Le catalogue, dans lequel la statuette est reproduite, est toutefois un témoignage précieux pour comprendre dans quels discours elle s'intégrait. La section dédiée à la Préhistoire fut placée sous le commissariat de Guy de Beauchêne, géologue africaniste. Dans son texte d'introduction, il insiste sur le caractère religieux des productions artistiques préhistoriques ${ }^{3}$. Une erreur présente dans la notice consacrée à la Vénus de Lespugue atteste des confusions autour de son numéro d'inventaire. Il est en effet précisé qu'elle fut donnée en 1926 au Laboratoire d'Anthropologie du Muséum par le docteur et la comtesse de Saint-Périer, enregistrée sous le numéro $1926.5^{4}$. Un autre numéro d'inventaire, dont l'usage semble beaucoup plus limité puisque c'est la seule apparition constatée, est mentionné également pour le musée de l'Homme: le numéro 38.189. Ce numéro est d'autant plus énigmatique qu'il ne s'intègre pas à la nomenclature tripartite imposée par Rivet et son sous-directeur Georges-Henri Rivière (1897-1985) dès 1928.

Cette même année 1965 est publié le premier volume de la collection Arts et grandes civilisations par les éditions Citadelle \& Mazenod, consacré à la Préhistoire de l'art occidental. Cette nouvelle collection est à mettre en lien avec l'engouement pour les albums et autres livres d'art qui permettent le rassemblement d'un grand nombre d'œuvres de même style pour un nouveau regard sur elles. La direction de ce volume est confiée à André Leroi-Gourhan qui fait le choix éditorial de mettre en valeur la dimension artistique de toutes les productions des premiers temps paléolithiques. La Vénus de Lespugue y est qualifiée de chef d'œuvre de la figure féminine ${ }^{5}$. Les théories formelles, autour du cercle abdomino-pelvien et du losange structural, définies par Leroi-

\footnotetext{
${ }^{1}$ Cf. F. Grognet, 1938-2009: un voyage dans les galeries du musée de l'Homme.

${ }^{2}$ Communication personnelle de Philippe Mennecier, le 4 mai 2016.

${ }^{3}$ Cf. G. de Beauchêne, Préhistoire, p. 30.

${ }^{4}$ Cf. G. de Beauchêne, Préhistoire, p. 32.

${ }^{5}$ Cf. A. Leroi-Gourhan, Préhistoire de l'art occidental, p. 113.
} 
Gourhan pour l'ensemble des statuettes paléolithiques tiennent une place importante et permettent une nouvelle lecture stylistique de ces objets.

Avec l'arrivée de Henri Vallois (1889-1981), succédant à Rivet à la chaire d'étude des hommes actuels et des hommes fossiles en 1954 et plus vivement intéressé par le versant biologique de l'anthropologie, se pose la question de la place de la préhistoire au musée de l'Homme. En 1962 est créée une chaire dédiée, dont le premier titulaire est Lionel Balout (1907-1992). Soutenant une approche tournée vers le concept et le geste créateur en vue de faire apparaître des typologies, Balout rejette tout vocabulaire esthétisant au profit d'une grammaire du lithique qui peut paraître aride et désincarnée ${ }^{1}$. Toutefois, cette chaire n'est pas accueillie au musée de l'Homme mais dans les murs de l'IPH'. Les collections du département de Préhistoire restent jusqu'en 1969 sous la seule tutelle du laboratoire d'anthropologie. Un plus grand dynamisme est généré avec la nomination d'Henry de Lumley (né en 1934) en 1980. La première grande exposition du laboratoire de Préhistoire au musée de l'Homme est proposée d'octobre 1984 à décembre 1985 et s'intitule Art et civilisations des chasseurs de la Préhistoire, $34000-8000$ ans avant J.-C. Le catalogue publié mentionne les circonstances de découverte de la statuette, la description donnée par le comte de Saint-Périer en 1922 dans L'Anthropologie et propose l'hypothèse amusante formulée par George-Henri Luquet à propos du pagne qui serait une erreur du sculpteur ayant voulu figurer une chevelure ${ }^{3}$. Les conventions stylistiques mises en évidence par André Leroi-Gourhan sont également rappelées. Selon Odile Romain, c'est la première fois que l'original de la Vénus de Lespugue était exposée dans les murs du musée, dans la limite de l'exposition temporaire. Par la suite, cette manifestation fut présentée à l'American Museum of Natural History de New York. Marie Perpère, ancienne membre du laboratoire de Préhistoire du musée de l'Homme, eut alors à convoyer la Vénus de Lespugue jusqu'à New York en 1986, accompagnée d'un responsable du musée américain. Son récit de l'évènement traduit la grande préciosité de la Vénus et les efforts déployés pour la protéger. Après une traversée de l'Atlantique en avion-cargo, pendant laquelle Marie Perpère eut à porter le petit coffret de bois, tendu de satin blanc, qui avait été fabriqué pour le transport de la Vénus ${ }^{4}$, elle fut conduite au milieu de la nuit au musée dans une limousine encadrée de policiers armés en motos. Une fois arrivée sur les lieux, la Vénus fut immédiatement à nouveau enfermée dans un coffre avant sa mise en vitrine finale.

C'est finalement dans un coffre que la Vénus de Lespugue aurait passé la plus grande partie de sa vie muséale. Objet en ivoire de mammouth et demandant donc des mesures de conservation adéquates, elle fait partie des objets les plus fragiles des collections du MNHN. En raison de sa forte valeur

\footnotetext{
${ }^{1}$ Cf. A. Hurel, Quelle place pour la Préhistoire au Palais du Trocadéro?, p. 117.

${ }^{2}$ Cf. A. Hurel, Quelle place pour la Préhistoire au Palais du Trocadéro?, p. 116.

${ }^{3}$ Cf. Art et civilisations des chasseurs de la Préhistoire, pp. 122-125. Pour son hypothèse: G.-H. Luquet, Les Vénus paléolithiques.

${ }^{4}$ Courriel de Marie Perpère en date du 29 avril 2016.
} 
et de sa fragilité, Marie Perpère et Odile Romain signalent que l'original était conservé dans un coffre-fort dans le bureau de Paul Rivet puis des autres directeurs du musée de l'Homme, en compagnie des crânes de Cro-Magnon et de la Chapelle-aux-Saints ${ }^{1}$. Véronique Laborde reconnaît que la Vénus de Lespugue fut longtemps conservée dans un coffre-fort au sein des réserves, au moins depuis l'arrivée de cet agent au musée en 2004. Ce coffre blindé est situé dans une salle permettant une température et une hygrométrie constante dans laquelle sont également préservés les crânes mentionnés par Marie Perpère ${ }^{2}$.

\section{La Vénus de Lespugue aujourd'hui: la Joconde du nouveau musée de l'Homme}

À partir de 2001 et à la suite de la modification de ses statuts, le Muséum national d'Histoire naturelle entre en phase de restructuration. Celle-ci intervient en parallèle du projet du musée du quai Branly et va accélérer la

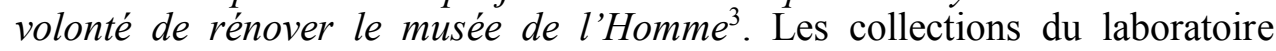
d'ethnologie, symboles du musée de l'Homme depuis son ouverture en 1938, déménagent à partir de mars 2003 pour l'essentiel vers le futur musée du quai Branly-Jacques Chirac, d'autres vers Marseille où est projeté un musée des Civilisations d'Europe et de Méditerranée (MuCEM). Le musée de l'Homme ferme ses portes le 23 mars 2009 et entame des travaux de rénovation qui durent plus de six ans. Une commission de rénovation est lancée en 2003 autour du préhistorien Jean-Pierre Mohen et vise à mieux connaître et comprendre le passé de l'institution, inventaire critique nécessaire à une nouvelle histoire ${ }^{4}$. Les résultats de cette commission, publiés sous le titre Le Nouveau Musée de l'Homme, ont servi de base à l'élaboration du Projet Scientifique et Culturel de l'établissement ${ }^{5}$. La reconversion du musée de l'Homme dut s'opérer autour des collections conservées par les laboratoires de préhistoire et d'anthropologie, et en adéquation avec la ligne directrice du MNHN. S'y développe ainsi une Histoire naturelle de l'Homme et histoire culturelle de la nature ${ }^{6}$. La préhistoire prend une place majeure dans cette visée, par son rapprochement avec l'ethnologie refondée et le nombre de préhistoriens œuvrant à la rénovation.

Le nouveau musée de l'Homme s'est construit avec la volonté de poursuivre l'œuvre de Paul Rivet dans la réunion des sciences de l'Homme pour leur développement, leur enseignement et leur diffusion à un plus large public. Le concept de musée-laboratoire est ainsi la pierre angulaire de sa renaissance. Toutefois, si les scientifiques régnaient en maîtres dans ce musée dans les années 1930, la naissance de nouveaux lieux de recherche a entraîné une nouvelle cartographie des savoirs. La fonction scientifique a progressivement

\footnotetext{
${ }^{1}$ Courriel de Marie Perpère en date du 29 avril 2016. Communication personnelle d'Odile Romain, le 4 avril 2016.

${ }^{2}$ Communication personnelle de Véronique Laborde, le 2 juillet 2016.

${ }^{3}$ M. Bertin, Penser soi et penser l'autre ..., p. 48.

${ }^{4}$ Musée de l'Homme, La Saga de l'Homme (épisode 1: l'homme exposé).

${ }^{5}$ Cf. Le Nouveau Musée de l'Homme, (éd.) J.-P. Mohen.

${ }^{6} \mathrm{Cf}$. S. Bahuchet, L'Homme indigeste?, p. 59.
} 
diminué dans les musées au profit de ses fonctions culturelle et politique ${ }^{1}$, créant aujourd'hui un véritable décalage entre ces établissements et la recherche. La structure interne du MNHN, partagée entre départements de recherche et départements de diffusion ${ }^{2}$, manifeste tout à fait cette réalité. Le musée de l'Homme est donc nominalement et administrativement dédié à la présentation au public et non à l'étude scientifique. Le retour au Trocadéro des laboratoires du MNHN dédiés à l'anthropologie et à la préhistoire est significatif et permet un rapprochement réel avec les équipes en charge de la muséographie, mais il nécessite un effort de collaboration entre les différents départements afin de conserver une dynamique de diffusion.

Pendant la durée du chantier, les collections sont restées invisibles au public. Certaines sont toutefois prêtées pour des expositions temporaires. C'est le cas de la Vénus de Lespugue qui est exposée par deux fois lors de manifestations de courte durée. Entre mars 2004 et janvier 2005 d'abord, elle est déplacée au sein de la Grande Galerie de l'Évolution au Jardin des Plantes pour Au Temps des mammouths. Elle y illustre les premières productions humaines réalisées en ivoire de mammouth ${ }^{3}$. Puis, en 2010, Véronique Laborde convoie la statuette jusqu'au muséum de Toulouse afin qu'elle soit présentée pour l'exposition Le Muséum de Toulouse et l'invention de la Préhistoire ${ }^{4}$. Dans le catalogue publié à l'occasion de cette manifestation, la Vénus de Lespugue est reproduite comme illustration en regard de la biographie du couple SaintPérier. L'exposition s'est en effet attachée à dresser le portrait de scientifiques et préhistoriens ayant réalisé des fouilles dans la région de Toulouse en mettant en valeur leurs découvertes. La Vénus de Lespugue fut également rendue visible par un autre biais avant la réouverture du musée de l'Homme. Au moment où la date d'ouverture du musée est fixée, une banderole en façade annonce son approche imminente et permet de refaire la promotion d'un musée qui semblait fermé à jamais. C'est la Vénus de Lespugue qui illustre cette affiche, incarnant à elle seule l'intégralité des collections conservées par le musée aux yeux du public. Elle est également omniprésente dans les dossiers de presse et documents de communication réalisés par le musée à la veille de son inauguration.

Le musée de l'Homme rouvre ses portes le 17 octobre 2015. Le parcours permanent mis en place s'articule en trois parties et tente de définir le propre de l'Homme: Qui sommes-nous?, D'où venons-nous?, Où allons-nous? Selon Agnès Parent, cheffe de projet de la conception-réalisation de la Galerie de l'Homme, une muséographie d'idées est adoptée incarnant des propos et des discours forts, tout en mettant en valeur de beaux objets, choisis avec soin ${ }^{5}$. La mise en espace a été conçue avec l'agence d'architecture parisienne Zen $+\mathrm{dCo}$ dirigée par Zette Cazalas. De nouveaux objets phares ont dû être définis dont la Vénus de Lespugue fait partie. Le guide de visite proposé au public à l'entrée

\footnotetext{
${ }^{1}$ Cf. F. Grognet, La Réinvention du Musée de l'Homme ..., p. 65.

${ }^{2} \mathrm{Cf}$. Muséum national d'Histoire naturelle, organisation.

${ }^{3}$ Cf. [Exposition] Au Temps des mammouths.

${ }^{4}$ Cf. Le Muséum de Toulouse et l'invention de la Préhistoire.

${ }^{5}$ Cf. A. Parent, Le nouveau musée de l'Homme.
} 
du musée intègre ces objets à ne pas manquer. En lien avec l'engouement pour les ventes de souvenirs au sein des musées, des moulages de la Vénus de Lespugue sont proposés à la vente à la boutique, dans une version reconstituée en résine ${ }^{1}$.

Une section complète ( $D$ 'où venons-nous?) est consacrée à la préhistoire, dans le but de mettre en valeur les fondements de notre civilisation et les liens que l'être humain actuel entretient avec ses premiers ancêtres. C'est dans cette partie qu'est exposée la Vénus de Lespugue, au sein d'une alcôve réservée à l'art mobilier préhistorique, les plus beaux objets du musée selon Agnès Parent ${ }^{2}$. C'est la véritable Vénus qui est présentée au public, malgré les réticences formulées par certains chercheurs du musée de voir s'éloigner des objets qui leur étaient autrefois facilement accessibles pour mener leurs recherches. Le départ d'une partie des collections de l'ancien musée de l'Homme, vécue comme un véritable traumatisme par les membres du musée, a révélé un grand attachement aux objets et permet de comprendre également ces oppositions (cf. Illustration $n^{\circ} 2$ et Illustration $n^{\circ} 3$ ).

Cette alcôve est qualifiée de nouvelle Salle du Trésor par l'historienne Alice Conklin ${ }^{3}$. Ce nom apparait d'ailleurs textuellement à l'entrée de l'espace depuis le printemps 2018. La Vénus de Lespugue est présentée dans une vitrine centrale, permettant de tourner autour et de la voir sous ces divers angles, maintenue à hauteur d'yeux par une tige métallique. Son extrême fragilité et sa préciosité obligent à l'enserrer dans une vitrine blindée. Ce mode de présentation tient tout du reliquaire, permettant à la fois de protéger et mettre en valeur l'objet. La lumière tamisée et l'espace resserré tendent à créer un effet de rencontre, afin de provoquer l'émotion du visiteur. Dans son nouvel écrin, elle est présentée hors du temps, flottant au centre de la vitrine, telle une apparition. Le parti-pris est éminemment esthétique. Dans cette volonté, le cartel se limite à présenter le contexte de découverte en fouilles de la Vénus et une description reprenant l'hypothèse d'une double figure par retournement de la statuette émise par Yves Coppens ${ }^{4}$, membre du Comité d'Orientation du musée de l'Homme. Sa datation approximative, 23000 BP, est donnée, mais semble une abstraction en regard de la contemplation offerte au regard. Son parcours entre les différents laboratoires du MNHN et les théories dont elle fut l'objet ne sont également pas indiqués. Il est intéressant de noter qu'aucun nom d'individu n'apparaît sur ce cartel, pas même le comte de Saint-Périer. Le catalogue publié en parallèle de l'inauguration de la Galerie de l'Homme mentionne une utilisation inconnue ${ }^{5}$.

\footnotetext{
${ }^{1}$ Réunion des musées nationaux - Grand Palais (RMN-GP), Moulage en résine de la Vénus de Lespugue.

${ }^{2}$ Communication personnelle d'Agnès Parent, le 6 janvier 2015. Entretien repris in: M. Bertin, Penser soi et penser l'autre ..., pp. 31-34.

${ }^{3}$ Cf. A. Conklin, Exposer l'humanité, p. 479.

${ }^{4} \mathrm{Cf}$. Y. Coppens, L'Ambiguïté des doubles Vénus du Gravettien de France.

${ }^{5}$ Cf. Le Musée de l'Homme: itinéraire, p. 89.
} 
5. Conclusion: Préhistoire en crise ou antagonisme de la science au musée?

La préhistoire a progressivement gagné droit de cité au Trocadéro, mais de manière imparfaite. La réunion des collections et des laboratoires a marqué une étape importante sans entièrement dissoudre la difficile situation actuelle des sciences au sein des musées. Actuellement, la science ne se fait plus dans les musées: l'université est devenue le lieu de recherche privilégié pour les sciences humaines. L'éloignement des chercheurs vis-à-vis des collections muséales pour leur étude est le fruit d'un double mouvement, en raison à la fois de l'évolution des disciplines et des centres d'études, mais également de la création de nouveaux corps de métiers plus spécialisés en sein desdites institutions muséales. La dichotomie entre collections d'études, dont le statut reste flou, et collections d'objets à exposer entérine cette réalité. La note-circulaire du 19 juillet 2012 relative à la problématique des matériels d'étude et à la méthodologie préalable à l'affectation de certains de ces bien aux collections des musées de France vise particulièrement le matériel de fouilles ${ }^{1}$. Bien que la Vénus de Lespugue n'ait jamais été intégrée au sein des collections d'études, elle est devenue chef-d'œuvre et a été écartée des mains des chercheurs en faveur des yeux des visiteurs par sa mise en vitrine. Objet unique tant dans les collections du musée de l'Homme que pour l'ensemble du patrimoine mondial, la Vénus constitue un élément d'attraction indéniable pour les visiteurs du musée. Le quasi-culte de l'authenticité dans le domaine muséologique et la considération pour la vraie chose $e^{2}$ sont autant de raisons qui expliquent le choix de présenter l'original de la Vénus.

Si elle s'annonce comme la Joconde du musée de l'Homme, sa mise en exposition actuelle relève spatialement, et presque conceptuellement, de l'impasse ${ }^{3}$. Des études de public non publiées commandées par Agnès Parent au lendemain de la réouverture du musée prouvent qu'un certain nombre de visiteurs passent d'ailleurs à côté de cette salle sans la voir ${ }^{4}$. Ces études ont mené à des indications plus marquées de l'espace d'exposition à l'aide d'un autocollant géant sur le mur représentant la Vénus de Lespugue de dos. Le cartel qui lui est associé, au même titre que le catalogue édité sur la Galerie de l'Homme par le musée ${ }^{5}$, n'apporte que des informations très parcellaires. Le musée de l'Homme qui se veut un lieu de débats et de partage d'idées peine ici à générer une dynamique. Le musée semble être resté à l'écart des dernières études et propositions sur les Vénus paléolithiques. Les interprétations féministes de Catherine Hodge Mc Cold et Leroy Mc Dermott qui émettent l'hypothèse d'une représentation des femmes par elles-mêmes en regardant

${ }^{1}$ Ministère de la Culture et de la Communication, Direction générale du patrimoine, Service des musées de France, Note-circulaire du 19 juillet 2012 relative à la problématique des matériels d'étude et à la méthodologie préalable à l'affectation des certains de ces biens aux collections des musées de France, Paris 2012.

${ }^{2}$ Cf. D. Cameron, Un point de vue ..., p. 261: Les vraies choses du langage muséal sont des choses que nous présentons telles qu'elles sont et non comme des modèles, des images ou des représentations de quelque chose d'autre.

${ }^{3}$ Communication personnelle d'Arnaud Hurel, le 26 avril 2016.

${ }^{4}$ Communication personnelle d'Agnès Parent, le 4 décembre 2015.

${ }^{5}$ Cf. Le Musée de l'Homme: itinéraire. 
leurs propres corps ${ }^{1}$ ne figurent par exemple pas dans les documents diffusés. Dans le cas de la Vénus de Lespugue, l'esthétique prime sur la connaissance. Par sa muséographie, son programme scientifique et ses choix d'exposition, le musée de l'Homme illustre une forme de fétichisation ${ }^{2}$ des objets scientifiques, fruit d'un inexorable mouvement d'esthétisation de la science ${ }^{3}$. Celui-ci ne vient que trop parachever le constat de la difficile alliance de la science, sans cesse dynamique, et du musée, statique et amené à durer ${ }^{4}$.

\section{Bibliographie}

Art et civilisations des chasseurs de la Préhistoire, $34000-8000$ ans avant J.C., Muséum national d'Histoire naturelle, Paris 1984.

Bahuchet S., L'Homme indigeste? Mort et transfiguration du musée de l'Homme in: Le Musée cannibale, (éd.) M.-O. Gonseth, J. Hainard \& R. Kaehr, Musée d'ethnographie, Neuchâtel 2002, pp. 59-84.

Beauchêne de G., Préhistoire in: Chefs d'œuvre du musée de l'Homme, Caisse des Monuments Historiques, Paris 1965, pp. 27-37.

Bertin M., Penser soi et penser l'autre à travers les dispositifs de présentation du Musée de l'Homme et leur évolution, mémoire de diplôme en muséologie, sous la direction de C. Hurley-Griener, École du Louvre, Paris 2015.

Bertin M., Redéfinir les objets scientifiques en chefs d'œuvre: vie bien vécue ou contre-sens?, mémoire de master en muséologie, sous la direction de C. Hurley-Griener, École du Louvre, Paris 2016.

Bonnot T., L'Attachement aux choses, CNRS, Paris 2014.

Bonnot T., La Vie des objets, Éditions de la Maison des sciences de l'homme, Paris 2002.

Boule M., Les Hommes fossiles. Eléments de paléontologie humaine, édition par Henri Vallois, Masson et $\mathrm{C}^{\mathrm{ie}}$, Paris 1945.

Bourrillon R., Les Représentations humaines sexuées dans l'art du Paléolithique supérieure européen: diversité, réminiscences et permanences, thèse en archéologie préhistorique, sous la direction de M. Barbaza, Université de Toulouse II-Le Mirail, Toulouse 2009.

Cameron D., Un point de vue: le musée considéré comme un système de communication et l'implication de ce système dans les programmes éducatifs muséaux in: Vagues. Une anthologie de la nouvelle muséologie, (éd.) A. Desvallées, Savigny-le-Temple, MNES 1992, pp. 259-270.

Chefs d'œuvre du musée de l'Homme, Caisse des Monuments Historiques, Paris 1965.

Christy H. \& Lartet É., Sur des figures d'animaux gravées ou sculptées et autres produits d'art et d'industries rapportables aux temps primordiaux de la période humaine in: Revue archéologique 9, 1864, pp. 233-267. 
Clifford J., Malaise dans la culture. L'ethnographie, la littérature et l'art au $X X^{e}$ siècle, trad. M. $-\mathrm{A}$. Sichère, École nationale des Beaux-Arts, Paris 1996.

Conklin A., Exposer l'humanité. Race, ethnologie et empire en France (18501950), Publications scientifiques du Museum national d'Histoire naturelle, Paris 2015.

Coppens Y., L’Ambiguité des doubles Vénus du Gravettien de France in: Comptes-rendus des séances de l'Académie des Inscriptions \& BellesLettres 133-3, 1989, pp. 566-571.

Crémière C., La Science au musée. L'anatomie comparée au Jardin du Roi puis au Muséum national d'Histoire naturelle de Paris, 1745-1898, thèse de doctorat en muséologie, sous la direction de M. Drouin \& M. Van Praët, Muséum national d'Histoire naturelle, Paris 2004.

Delporte H., L'Image de la femme dans l'art préhistorique, Picard, Paris 1979.

[Exposition] Au Temps des mammouths [en ligne: http://mammouths.mnhn.fr/ frameset.htm, dernière consultation le 25 août 2018].

Foucher P. \& San Juan C., Les Industries solutréennes de l'abri des Harpons et de la Grotte des Rideaux (Lespugue, 31). Collections Saint-Périer des musées de Lespugue et de Saint-Gaudens in: Bulletin de la Société préhistorique Ariège-Pyrénées 4, 2001, pp. 27-33.

Ginzburg C., Signes, traces, pistes in: Le Débat n6, 1980, pp. 3-44.

Grognet F., Objets de musée, n'avez-vous donc qu'une vie? in: Gradhiva ${ }^{\circ} 2$, 2005, pp. 49-63.

Grognet F., Le concept de musée: la patrimonialisation de la culture des «autres» d'une rive à l'autre, du Trocadéro à Branly, histoire de métamorphose, thèse de doctorat en anthropologie, sous la direction de J. Jamin, EHESS, Paris 2009.

Grognet F., La Réinvention du Musée de l'Homme au regard des métamorphoses passées du Trocadéro in: Les Musées d'ethnologie: culture, politique et changement institutionnel, (éd.) C. Mazé, F. Poulard \& C. Ventura, CTHS, Paris 2013, pp. 37-70.

Grognet F., 1938-2009: un voyage dans les galeries du musée de l'Homme in: Le musée de l'Homme. Histoire d'un musée laboratoire, (éd.) C. Blanckaert, Artlys \& Museum national d'histoire naturelle, Paris 2015, pp. 176-205.

Hodge McCold C. \& McDermott L., Toward decolonizing gender female vision in the Upper Paleolithic in: American Anthropologists 98, 2/1996, pp. 319 326.

Hurel A., Des Bushmen en Europe? Les Vénus paléolithiques et les «négroïdes» de Grimaldi dans la construction de la préhistoire française in: La Vénus Hottentote entre Barnum et Muséum, (éd.) C. Blanckaert, Muséum national d'Histoire naturelle, Paris 2013, pp. 291-363.

Hurel A., Faire entrer la Préhistoire au musée in: Le Muséum de Toulouse et l'invention de la Préhistoire, (éd.) F. Bon, S. Dubois \& M.-D. Labails, Éditions du Muséum de Toulouse, Toulouse 2010, pp. 154-167.

Hurel A., Les Préhistoriques sous l'œil des naturalistes. Les hommes fossiles, entre le Muséum et l'Institut de Paléontologie humaine in: Penser, classer, 
administrer: pour une histoire croisée des collections scientifiques, (éd.) B. Daugeron \& A. Le Goff, Muséum national d'Histoire naturelle, Paris 2014, pp. 17-38.

Hurel A., Quelle place pour la Préhistoire au Palais du Trocadéro? in: Le musée de l'Homme. Histoire d'un musée laboratoire, (éd.) C. Blanckaert, Museum national d'histoire naturelle, Paris 2015, pp. 101-123.

Hurel A., Aux marches du Palais, la place de la Préhistoire au Trocadéro, communication lors du colloque Paul Rivet: un pont entre deux rives (1920-1950), musée du quai Branly - Jacques Chirac et musée de l'Homme, 14 décembre 2017.

Journal officiel de la République française, lois et décrets, en date du 15 octobre 1941 [en ligne: http://gallica.bnf.fr/ark:/12148/bpt6k96339501/f2, dernière consultation le 25 août 2018].

Laurière C., Paul Rivet (1876-1958), le savant et le politique, thèse de doctorat en anthropologie, sous la direction de J. Jamin, EHESS, Paris 2006.

L'Estoile de B., Le Goût des Autres. De l'exposition coloniale aux Arts premiers, Flammarion, Paris 2010.

Levi G., On Microhistory in: New Perspectives on Historical Writing, (éd.) P. Burke, Polity Press, Cambridge 2001, pp. 97-119.

Leroi-Gourhan A., Préhistoire de l'art occidental, Citadelles \& Mazenod, Paris 1995.

Luquet G.-H., Les Vénus paléolithiques in: Journal de psychologie normale et pathologique 31, 5-6/1934, pp. 429-460.

Ministère de la Culture et de la Communication, Direction générale du patrimoine, Service des musées de France, Note-circulaire du 19 juillet 2012 relative à la problématique des matériels d'étude et à la méthodologie préalable à l'affectation des certains de ces biens aux collections des musées de France, 2012.

Musée de l'Homme, La Saga de l'Homme (épisode 1: l'homme exposé), s. d. [en ligne: http://www.museedelhomme.fr/fr/musee/expositions-passees/ saga-homme-episode-1-homme-expose, dernière consultation le 25 août 2018].

Muséum national d'Histoire naturelle, organisation [en ligne: https://www. mnhn.fr/fr/organisation, dernière consultation le 25 août 2018].

La Préhistoire. Histoire et dictionnaire, (éd.) D. Vialou, Robert Laffont, Paris 2004.

Le Musée de l'Homme. Histoire d'un musée laboratoire, (éd.) C. Blanckaert, Artlys \& Museum national d'Histoire naturelle, Paris 2015.

Le Musée de l'Homme: itinéraire, Muséum national d'Histoire naturelle, Paris 2015.

Le Muséum de Toulouse et l'invention de la Préhistoire, (éd.) F. Bon, S. Dubois \& M.-D. Labails, Éditions du Muséum de Toulouse, Toulouse 2010.

Le Nouveau Musée de l'Homme, (éd.) J.-P. Mohen, Odile Jacob \& Museum national d'Histoire naturelle, Paris 2004.

Orliac R., L'invention de la préhistoire par les objets in: Les Nouvelles de l'archéologie $\mathrm{n}^{\circ} 129,2012$, pp. 13-20. 
Pales L., Les Ci-devant Vénus stéatopyges aurignaciennes in: Separata de Actas del Simposium de Arte rupestre, Santander 1972, pp. 217-261.

Parent A., Le nouveau Musée de l'Homme in: La Lettre de l'OCIM n²16, 2016, pp. 5-14.

Passemard L., Les Statuettes féminines paléolithiques dites Vénus stéatopyges, Librairie Tessier, Nîmes 1938.

Piette É., Races humaines de la période glyptique in: Bulletins de la Société d'anthropologie de Paris 5, IV ${ }^{\mathrm{e}}$ série, 1894, pp. 381-394.

Piette É., Station de Brassempouy et statuettes humaines de la période glyptique in: L'Anthropologie 6, 1895, pp. 129-151.

Poulot D., Patrimoine et musées. L'institution de la culture, Hachette, Paris 2014.

Réunion des musées nationaux - Grand Palais (RMN-GP), Moulage en résine de la Vénus de Lespugue, s. d. [en ligne: http://www.boutiquesdemusees.fr/ $\mathrm{fr} /$ boutiques/musee-de-lhomme/venus-de-lespugue/680.html, dernière consultation le 25 août 2018].

Saint-Périer de R., La Statuette féminine de Lespugue (Haute-Garonne) in: Bulletin de la Société préhistorique de France 21, 3/1924, pp. 81-84.

Saint-Périer de R., Statuette de femme découverte à Lespugue (Haute-Garonne) in: L'Anthropologie 32, 1922, pp. 361-381.

The Social Life of Things. Commodities in cultural perspective, (éd.) A. Appadurai, Cambridge University Press, Cambridge 1986.

Tilley C., Objectification in: Handbook of material culture, C. Tilley, W. Keane, S. Küchler, M. Rowlands \& P. Spyer, Sage Publications, London 2006, pp. 60-73.

Vénec-Peyré M.-T., La Difficile Naissance de la chaire de Paléontologie au Muséum national d'Histoire naturelle in: Penser, classer, administrer: pour une histoire croisée des collections scientifiques, (éd.) B. Daugeron \& A. Le Goff, Muséum national d'Histoire naturelle, Paris 2014, pp. 39-49.

Vialou D., Lespugue, femme ou Vénus? in: L'Art préhistorique $\mathrm{n}^{\circ} 209$, déc. 1995-jan. 1996, pp. 32-65. 


\section{Illustrations}

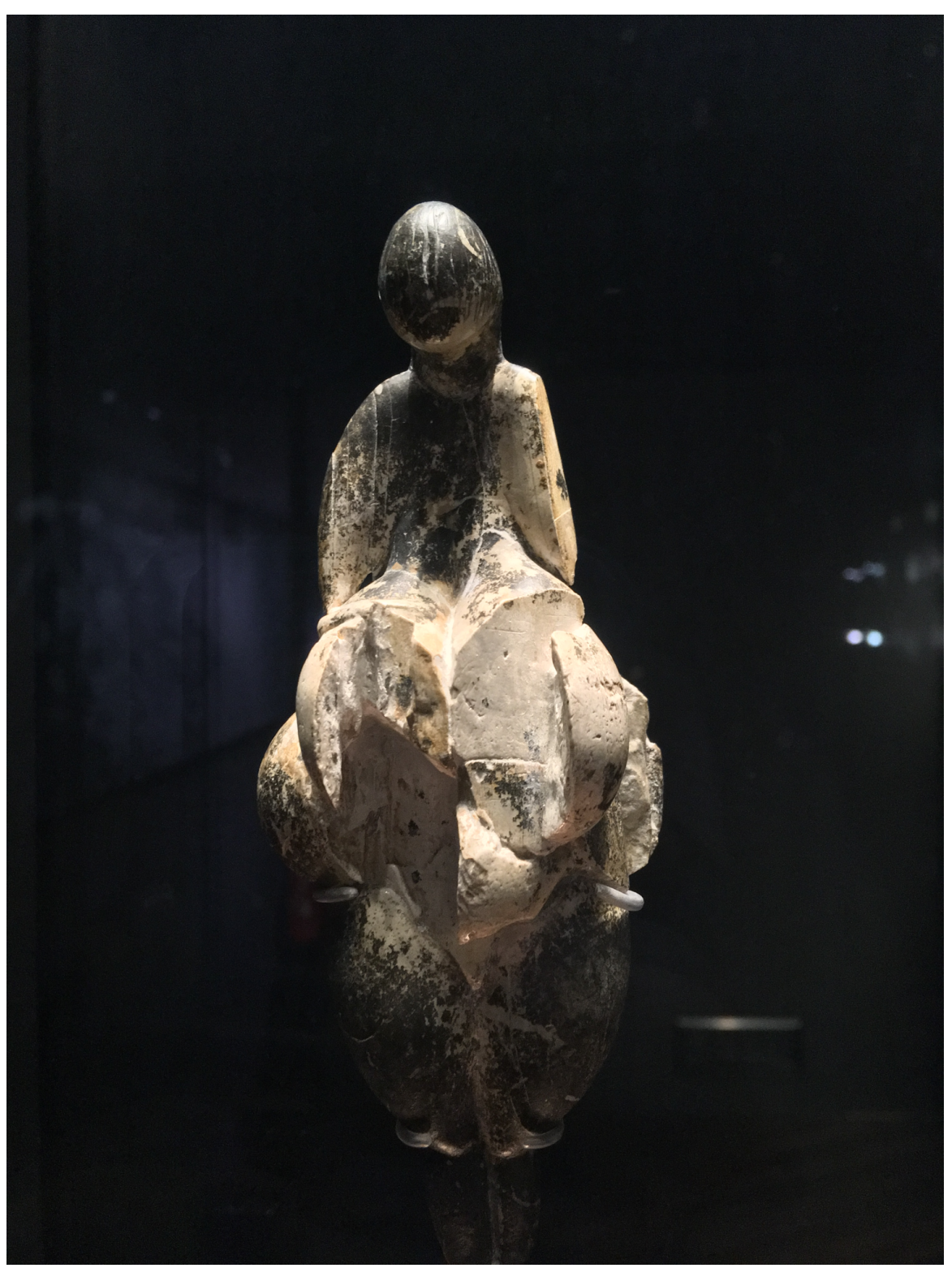

Illustration $n^{\circ} 1$ : Exposition actuelle de la Vénus de Lespugue au sein du musée de l'Homme, décembre 2017 [photographie personnelle]. 


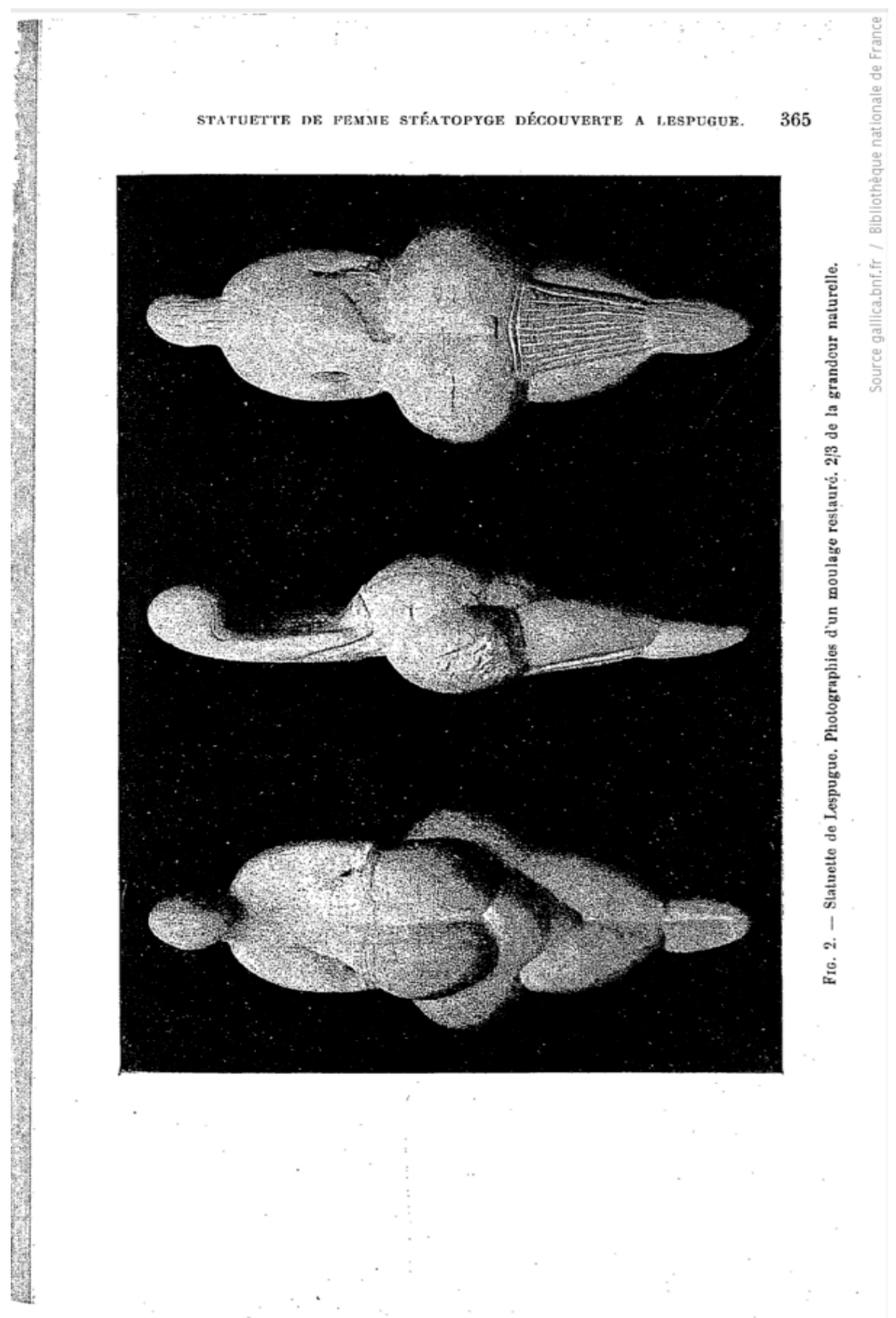

Illustration $\mathrm{n}^{\circ}$ 2: Statuette de Lespugue, photographie d'un moulage restauré au 2/3 de la grandeur naturelle in: R. de Saint-Périer, Statuette de femme découverte à Lespugue (Haute-Garonne) in: L'Anthropologie 32, 1922, p. 365 [disponible en ligne sur Gallica]. 


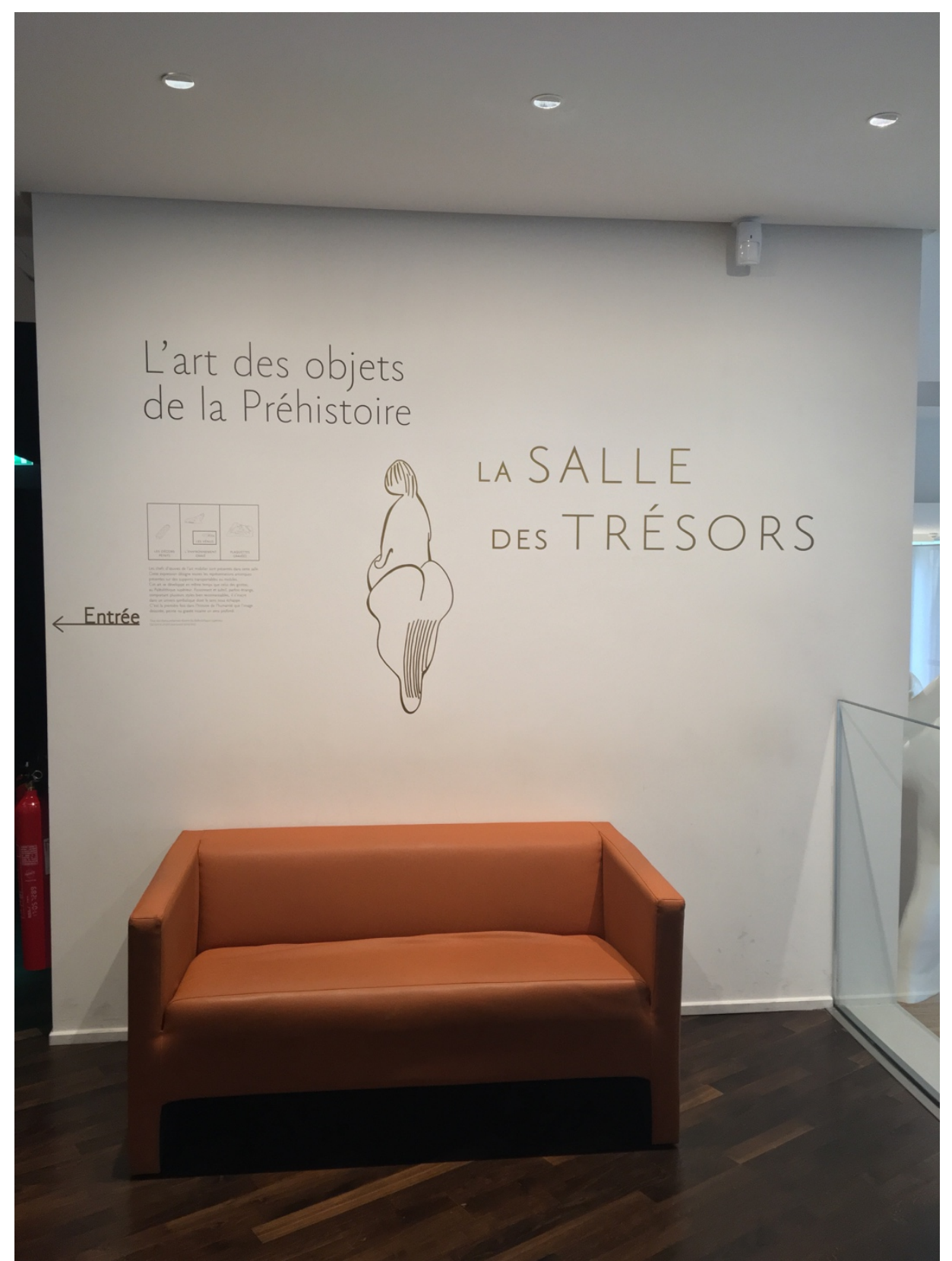

Illustration n`3: Entrée de La Salle des Trésors du musée de l’Homme, juin 2018 [photographie personnelle]. 